\title{
Study of the plasticity effect at the crack tip in Titanium by using thermal signal analysis
}

\author{
by F. Di Carolo*, R. De Finisi* D.Palumbo* J. M. Vasco-Olmo** ,F. A. Díaz ${ }^{* *}$, U. Galietti* \\ *Politecnico di Bari, Via Edoardo Orabona, 4, 70126 Bari, Italy \\ ** Departamento de Ingeniería Mecánica y Minera, University of Jaén, s/n, 23071 Jaén, Spain
}

\begin{abstract}
The fatigue crack growth is strictly related to the dissipated energy at the notch/crack tip. Among the different approaches developed, those focusing their attention on energy dissipated in the plastic area seems to be more promising in the evaluation of the crack growth behaviour and it can be used to describe the plastic work at the crack tip. In this work, an experimental approach was adopted in order to obtain thermographic parameters capable of describing the plastic work at the crack tip. In particular, the influence of tests parameters such as the loading frequency, has been investigated.
\end{abstract}

\section{Introduction}

The process of crack growth is affected by the energy dissipated at the notch/crack tip. In effect, in literature different analytical and numerical models [1-3] were proposed by researchers to describe as the plastic work affects the fatigue behaviour.

In recent years, researchers focused their attention on energy-based approaches [5-7] as the dissipated energy plays a key role in the crack growth behavior and it can be used to describe the plastic work at the crack tip [8-9]. The energy-based approach proposed firstly by Weertman [1], links the crack growth rate with the critical energy to create a unit surface area. Mazari et al., [3] starting from the Weertman's and Klingbeil's [2] approaches, developed a new model in which a similar Paris Law model was obtained between the crack growth and the heat dissipated per cycle.

In this experimental work thermoelastic and thermal parameters, capable of describing the plastic work at the crack tip, were used for studying fracture mechanics of pure titanium small scale samples. Moreover, the influence of loading frequency was investigated too.

\section{Theory}

The thermal signal analysis is performed by acquiring the thermal response of components and samples undergoing dynamical loads.

For a homogenous and isotropic material, the calibrated thermographic signal $\mathrm{T}$ during a fatigue test, can be written in the time domain as [10]:

$$
T(t)=T_{0}+a t+T_{1} \sin \left(\omega t+\Phi_{1}\right)+T_{2} \sin \left(2 \omega t+\Phi_{2}\right)
$$

The second term in Eq.1 represents the increase in mean temperature $T_{0}$, whose linear growth with the time is defined through the coefficients a which depends on the physic characteristics and geometry of the component. This term is affected by the heat transfer (conduction in the specimen or convection and radiance to the environment).

The application of a sinusoidal load with a sufficiently high frequency, make the heat transfer negligible and the Fourier harmonic components can be experimentally measured performing a lock-in analysis of the signal.

The third term in Eq. 1 is the thermoelastic component, varying at the same angular frequency as the load; $T_{1}$ and $\varphi_{1}$ are respectively the amplitude and phase of first harmonic amplitude component of Fourier. The phase $\Phi_{1}$ represent the delay between the load and the thermal response and under adiabatic conditions it is constant throughout the component but it varies if the adiabatic condition is no longer respected; this could be the case of high stress gradients, which lead to conduction effects [11,12] or heat generation due to local plasticity[13, 14].

The amplitude $T_{2}$ and phase $\Phi_{2}$ of the second Fourier harmonic components, are the result of two effects both occurring at the twice of the loading frequency: the thermoelastic response due to the second order effect $[15,16]$ and the temperature variation due to dissipation[17].

Referring to the amplitude, when local plasticization effects occur, the thermoelastic component is at least one order of magnitude smaller than the second and therefore it can be neglected, in this case, the measured $\mathrm{T}_{2}$ can be considered only related to the energy dissipation due to plasticization.

The capability of these parameters in fracture mechanics behaviour assessment was demonstrated by several authors $[8,10$.$] . In particular, the amplitude T_{2}$ allows the evaluation of the plastic and crack closure areas [8] while the phase shifts $\Phi 1$ [10] and $\Phi 2$ [8] are used to localise the crack tip [10]. 
In the present work, $T_{2} \Phi_{1}$ and $\Phi_{2}$ are used to study the influence of the loading frequency on the thermal footprint of the plastic area ahead the crack tip.

\section{Experimental campaign and Signal processing}

In this work, small scale compact tension specimens made of pure grade 2 Titanium were tested. The sample geometry is reported in figure1, while the material characteristics are reported in table 1

Table 1. Pure Titanium mechanical characteristics

\begin{tabular}{|c|c|}
\hline Young modulus, $\mathrm{E}$ & $105 \mathrm{GPa}$ \\
\hline Yield strength, $\sigma_{\mathrm{y}}$ & $390 \mathrm{MPa}$ \\
\hline Ultimate tensile strength, $\sigma_{u}$ & $448 \mathrm{MPa}$ \\
\hline
\end{tabular}

The tests were carried out by using a loading frame MTS model 370 with a $25 \mathrm{kN}$ of capacity (figure 1). According to ASTM E 647, the test procedure involved constant-force-amplitude load at stress ratio $R=0.1$. The sample was precracked by applying loading cycles with the frequency of $17 \mathrm{~Hz}$, a maximum load of $750 \mathrm{~N}$ and $\mathrm{R}=0.1$ until the crack did not reached a length of about $3 \mathrm{~mm}$ (after 21000 cycles). The thermographic acquisitions were performed every 2000 cycles with a maximum load of $400 \mathrm{~N}, \mathrm{R}=0.1$ and different loading frequencies, ranging between 8 and $23 \mathrm{~Hz}$. The experimental campaign characteristics are reported in table 2.

Table 2. Experimental campaign

\begin{tabular}{|c|c|c|c|c|}
\hline $\mathrm{N}^{\circ}$ of cycles & $\begin{array}{c}F_{\max } \\
{[N]}\end{array}$ & $\mathrm{R}$ & $\begin{array}{c}\text { frequency } \\
{[\mathrm{Hz}]}\end{array}$ & $\begin{array}{c}\mathrm{N}^{\circ} \text { of } \\
\text { acquisitions }\end{array}$ \\
\hline 23000 & \multirow{17}{*}{400} & \multirow{17}{*}{0.1} & 17 & 3 \\
\hline 25000 & & & 8 & 3 \\
\hline 27000 & & & 10 & 3 \\
\hline 29000 & & & 13 & 3 \\
\hline 31000 & & & 15 & 3 \\
\hline 33000 & & & 17 & 3 \\
\hline 35000 & & & 19 & 3 \\
\hline 37000 & & & 21 & 3 \\
\hline 39000 & & & 23 & 3 \\
\hline 41000 & & & 8 & 3 \\
\hline 43000 & & & 10 & 3 \\
\hline 45000 & & & 13 & 3 \\
\hline 47000 & & & 15 & 3 \\
\hline 49000 & & & 17 & 3 \\
\hline 51000 & & & 19 & 3 \\
\hline 53000 & & & 21 & 3 \\
\hline 55000 & & & 23 & 3 \\
\hline
\end{tabular}

Thermographic sequences were acquired during tests by adopting the cooled detector of FLIR IR X6540 SC IR camera at a frequency of $200 \mathrm{~Hz}$. A geometrical resolution of $0.0352 \mathrm{~mm} /$ pixel was adopted for the crack monitoring. Figure 1 shows the setup and equipment.

Referring to equation 1, a mathematical algorithm implemented in IRTA® software was used to extract pixel by pixel, the phase angle and the amplitude of the first and second Fourier harmonics of the thermoelastic signal. Data are calibrated in temperature, assuming for the black painted surface an emissivity of 0.95 .

To compare the minimum $\Phi_{1}$ and the maximum $T_{2}$, the $2^{\circ}$ percentile and the $98^{\circ}$ percentile were respectively evaluated in an area extracted around the crack tip.

In order to reduce the noise, $T_{2}$ and $\Phi_{2}$ maps where filtered using a Gaussian filter, while the data used to plot $T_{2}$ and $\Phi_{2}$ horizontal profiles have been smoothed by applying a moving-average filter with a radius of 5 pixels. 

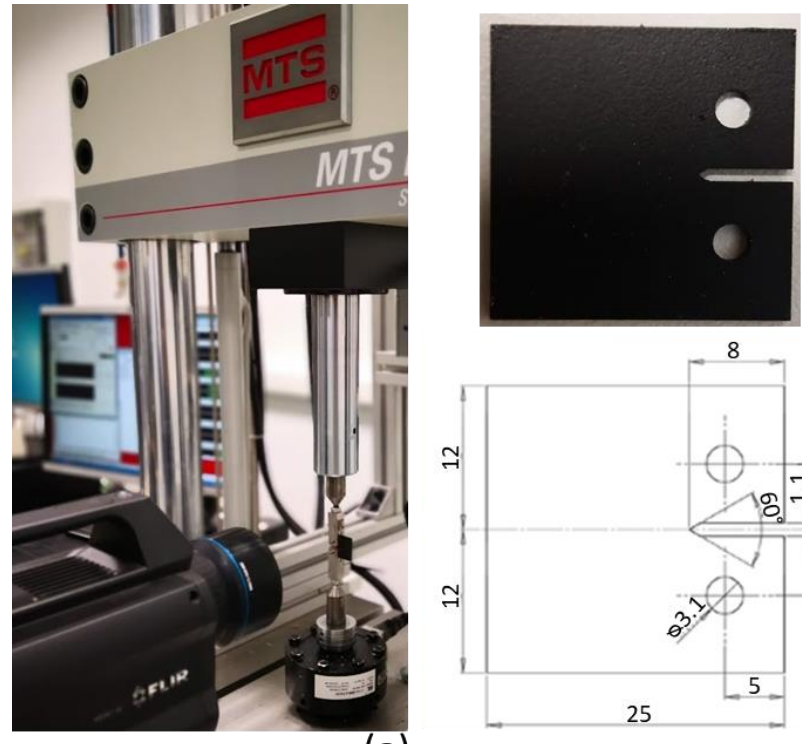

(b)

(a)

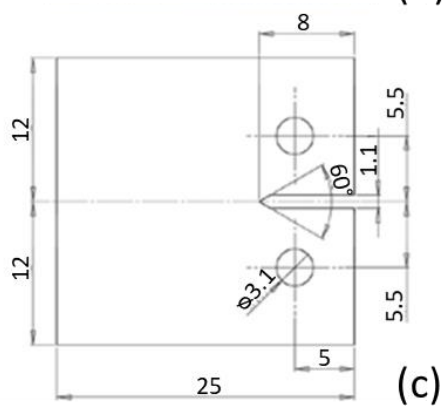

Fig. 1. (a) Setup and equipment. The sample face used for the TSA acquisitions was painted with a black mat spray (b). The measures are reported in $\mathrm{mm}(\mathrm{c})$

\section{Results and discussion}

Figure 2 shows the results in terms of maps of $\Phi 1, T 2$ and $\Phi 2$ at the crack tip for the loading frequency of $8 \mathrm{~Hz}, 17 \mathrm{~Hz}$ and $23 \mathrm{~Hz}$ of the area around the crack tip considered in this analysis.

The $\Phi_{1}$ and $\Phi_{2}$ data have been re-phased by subtracting the mean value evaluated in an area of the sample whose stress field is not affected by the crack during the loading.

For the two phases, the areas and the absolute value at the crack tip interested by the heat source due to the plasticity decrease when the frequency increases. The area and the absolute value of the amplitude $\mathrm{T}_{2}$, instead is lower at $17 \mathrm{~Hz}$. These trends are confirmed in In Figure 3, where the $2^{\circ}$ percentile of the phase signal and the $98^{\circ}$ percentile of the thermal signal are reported; while the former increases monotonically the latter stabilizes, being highly affected by the noise at around $15 \mathrm{~Hz}$.

In Figure 4, 5 and 6 are plotted the values of $\Phi_{1}, T_{2}$ and $\Phi_{2}$ along a horizontal profile parallel to the crack and passing for the crack tip for all the frequencies.

The comparison between the horizontal profiles gives information about the potential of the TSA in describing the area around the crack tip which is locally plasticizing. The $\Phi_{1}$ horizontal profiles show how the phase area affected by the nonadiabatic conditions (which imply a non-null phase value) decreases with the frequency but it seems to stabilize at roughly $17 \mathrm{~Hz}$.

From Figure 4, considering only the frequencies 17, 19, 21 and $23 \mathrm{~Hz}$ it is possible to infer the crack tip position between the pixels 520 and 521 (range of 1 pixel, corresponding to $0.0352 \mathrm{~mm}$ ), which is the point of sign-inversion of $\Phi_{1}$. At $8 \mathrm{~Hz}$ the point of sign inversion is shifted of 4 pixels $(0.14 \mathrm{~mm})$ on the right.

The point at which the first phase returns to zero can be related to the plastic zone extent but it is also influenced by conduction effects, which imply its variation with the loading frequency. In effect, the length of the negative section varies from 37 pixels $(1,3 \mathrm{~mm})$ at $23 \mathrm{~Hz}$ to 46 pixels $(1,62 \mathrm{~mm})$ at $15 \mathrm{~Hz}$ and to 65 pixels $(2.29 \mathrm{~mm})$ at $8 \mathrm{~Hz}$.

The comparison among the $T_{2}$ horizontal profiles (Figure 5) and the $\Phi_{1}$. horizontal profiles (Figure 4), shows how the pixels characterized by a negative $\Phi_{1}$ present an increase of the amplitude $T_{2}$, which is higher than the average value measured in the elastic area. The section on the right of the crack tip is interested by a maximum signal value, that the authors attribute to the crack closure [8], a dissipative phenomenon where the opposing faces of a crack remain in contact during the loading, occurring at twice of the loading frequency.

The crack closure effect is also evident in the $\Phi_{2}$ horizontal profiles on the right side of the red dashed line indicating the crack tip position in Figure 6. All the frequencies show a higher value at the crack interface.

The area ahead the crack tip is instead characterized by $\Phi_{2}$ negative values, however the phase variation is not high enough to be distinguished from noise. 

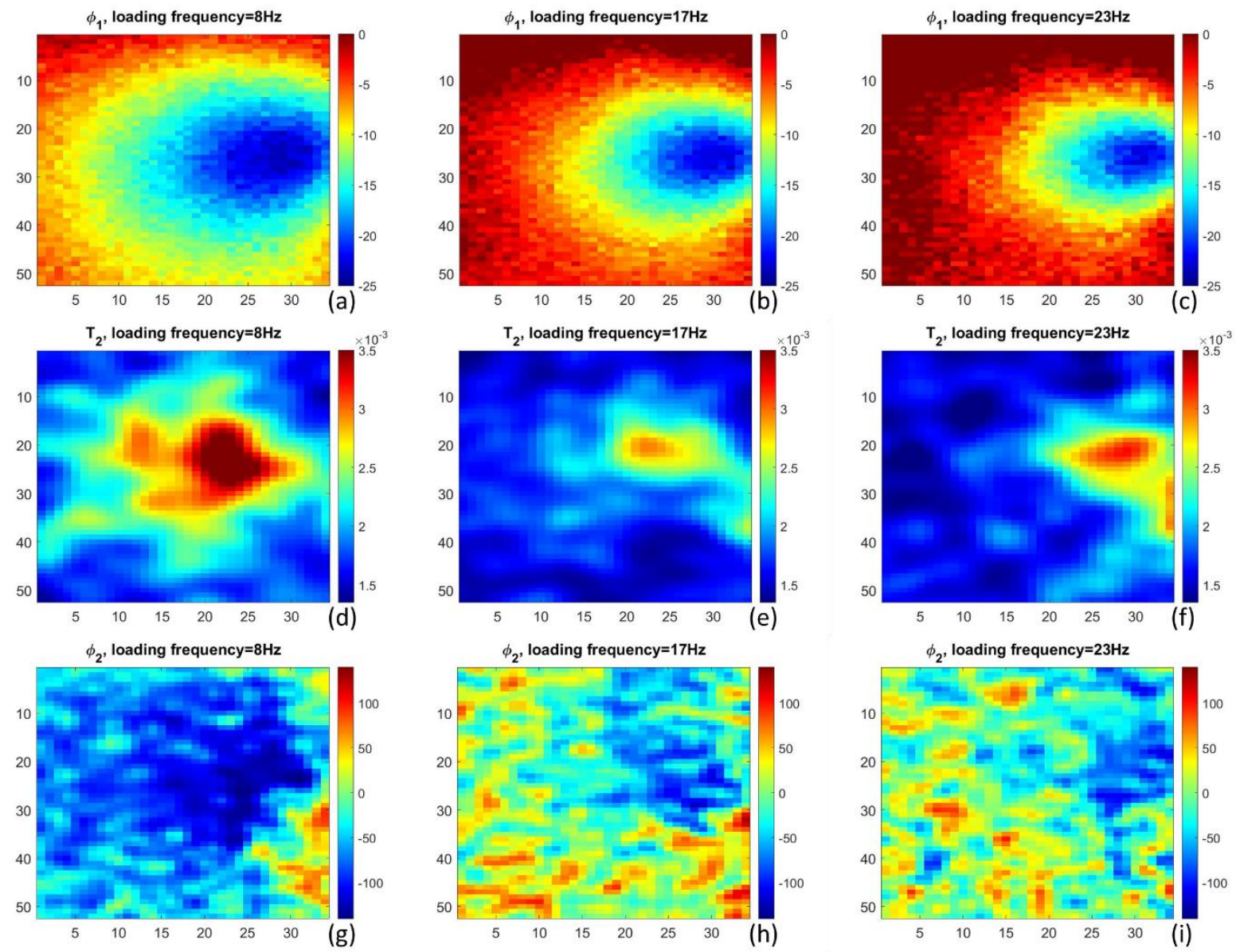

Fig. 2. $\Phi_{1}$ at the crack tip for the loading frequency of $8 \mathrm{~Hz}(\mathrm{a}), 17 \mathrm{~Hz}$ (b) and $23 \mathrm{~Hz}(c), T_{2}$ at the crack tip for the loading frequency of $8 \mathrm{~Hz}(d), 17 \mathrm{~Hz}(\mathrm{e})$ and $23 \mathrm{~Hz}(f)$ and $\Phi_{2}$ at the crack tip for the loading frequency of $8 \mathrm{~Hz}(\mathrm{~g}), 17$ $\mathrm{Hz}$ (h) and $23 \mathrm{~Hz}$ (i)
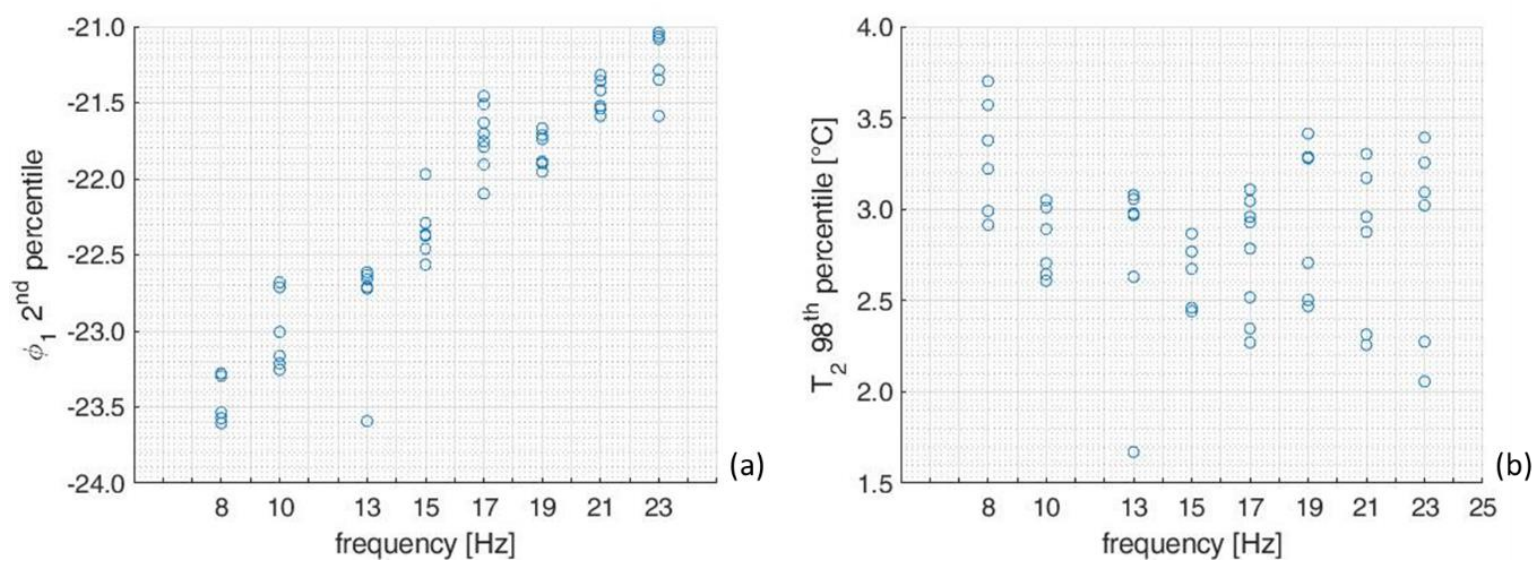

Fig. 3. a) $2^{\text {nd }}$ percentile of the phase signal and b) $98^{\text {th }}$ percentile of the $T_{2}$ as function of the loading frequency 


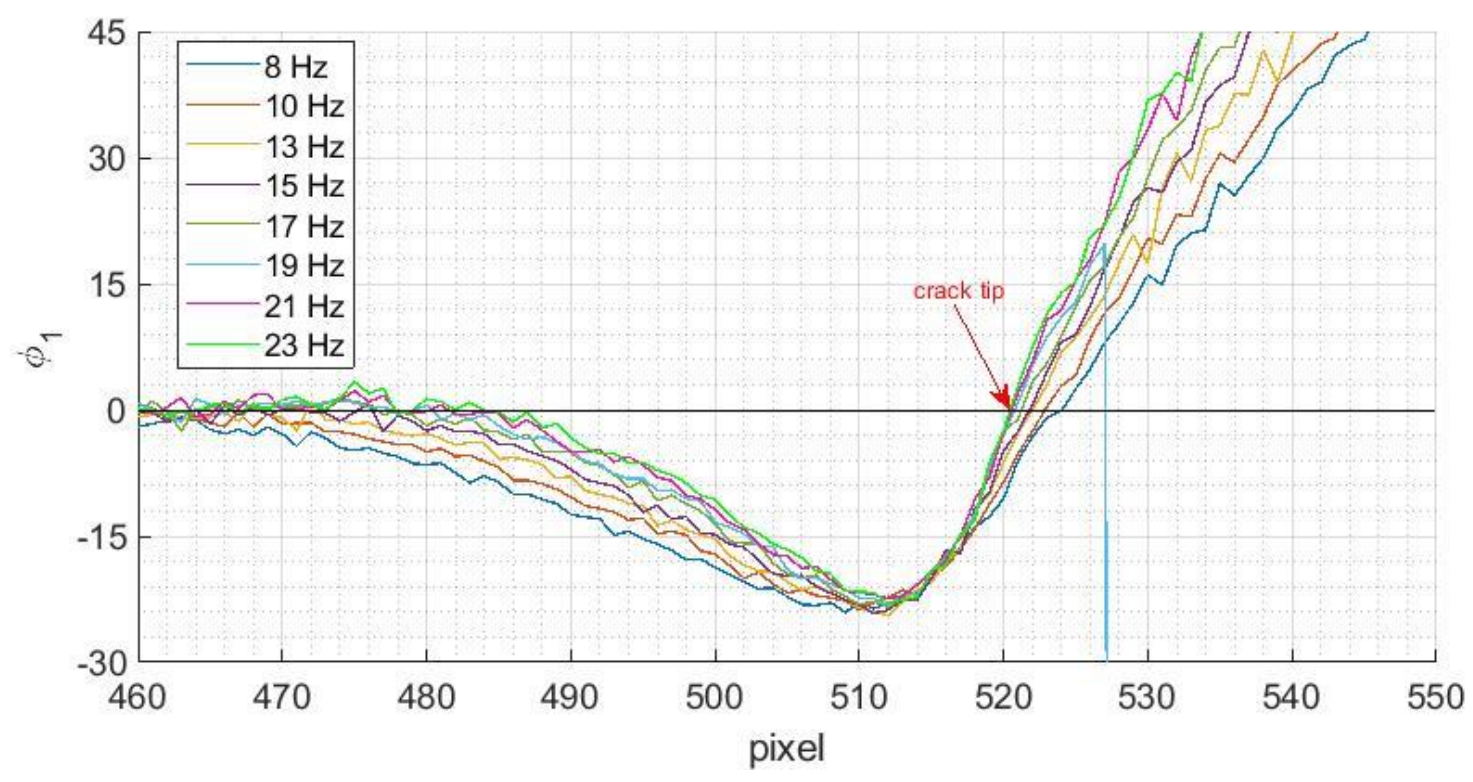

Fig. 4. $\Phi_{1}$ along a horizontal profile parallel to the crack and passing for the crack tip for all the tested loading frequencies

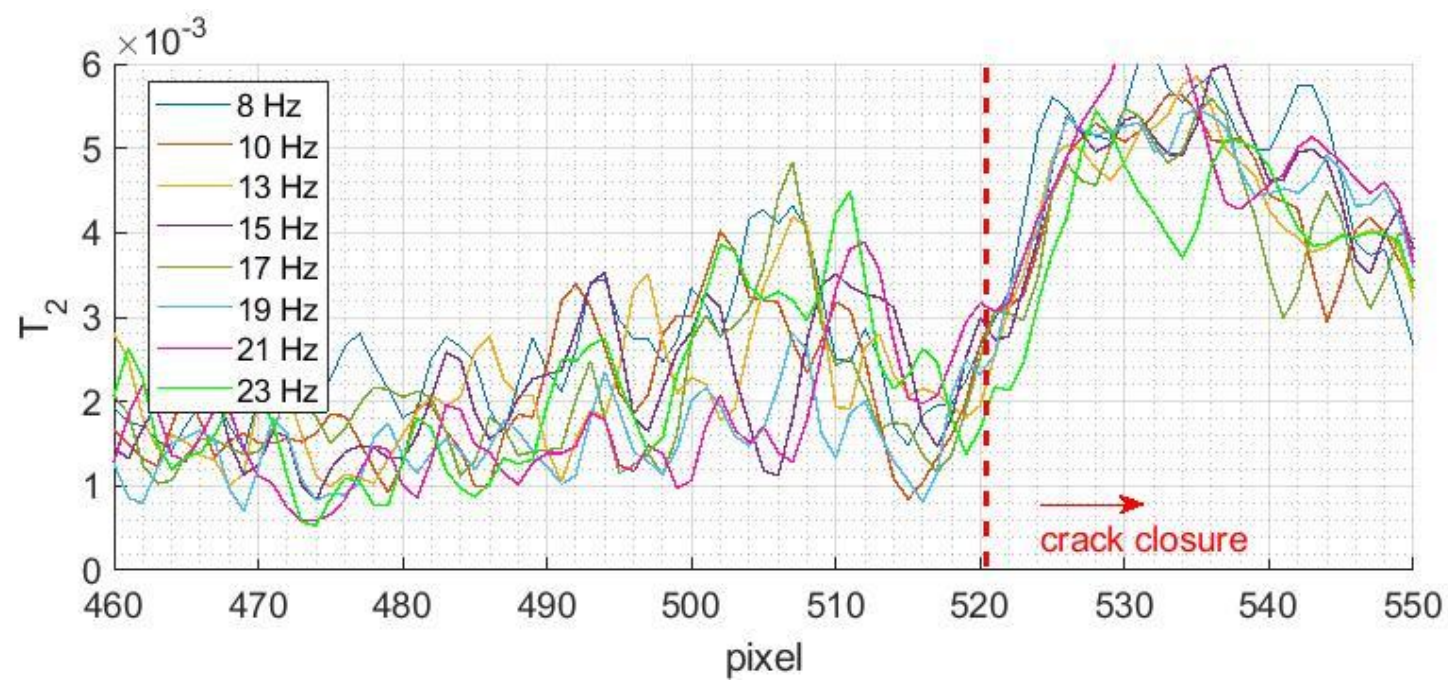

Fig. 5. $T_{2}$ along a horizontal profile parallel to the crack and passing for the crack tip for all the tested loading frequencies. The maximum reached at the left of the red dashed line is due to the crack closure effect. 


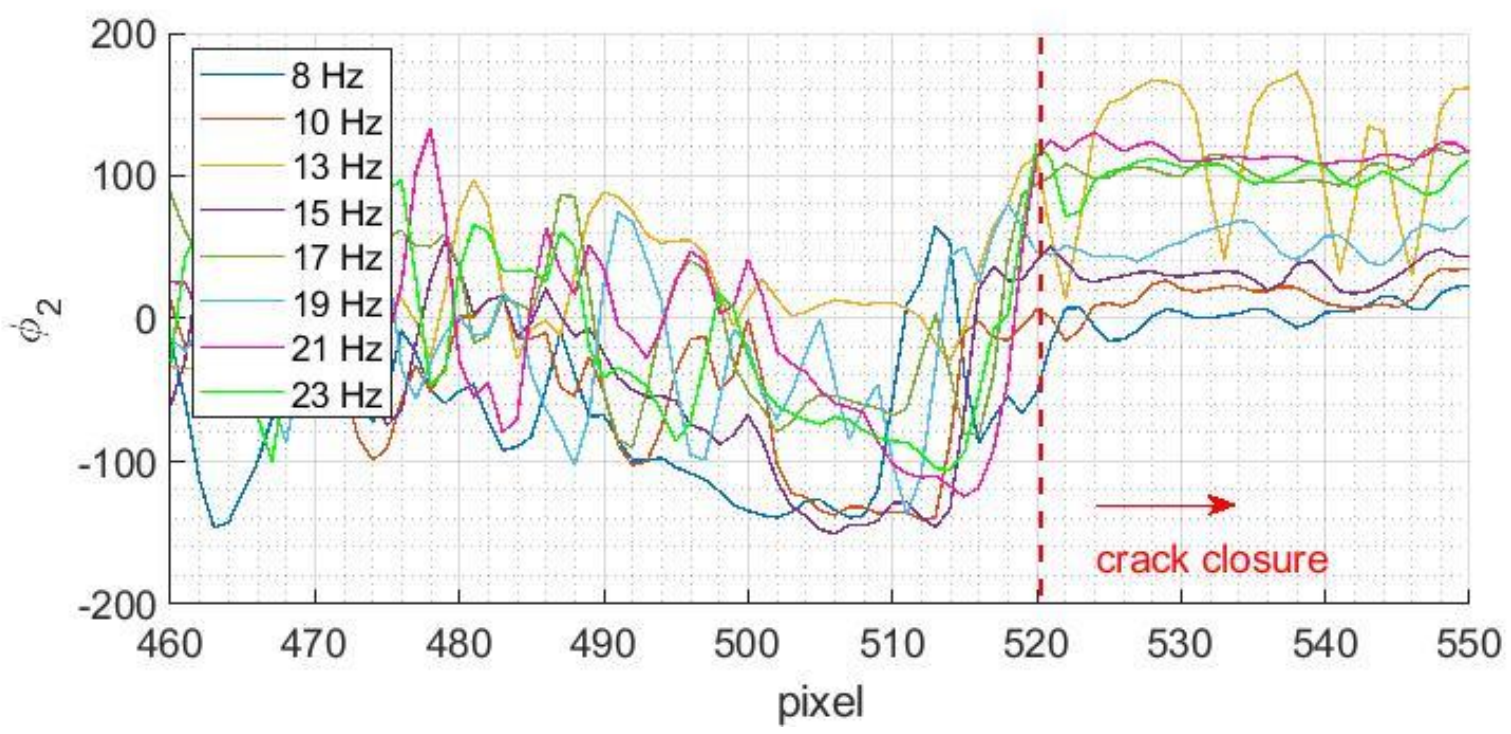

Fig. 6. $\Phi_{2}$ along a horizontal profile parallel to the crack and passing for the crack tip for all the tested loading frequencies. The maximum reached at the left of the red dashed line is due to the crack closure effect.

\section{Conclusions and Outlooks}

In this work the parameters $T_{2} \Phi_{1}$ and $\Phi_{2}$ which characterize the thermal response of a cracked Titanium CT samples under a cyclic load at different frequencies were studied in order to relate them to the plastic area ahead the crack tip.

The experimental tests showed the following results for the three parameters:

- $\quad$ The parameter $\Phi_{1}$ presents absolute values and an area of sign inversion which decrease with the frequency; However, the difference is smaller at the higher frequencies. In the frequency range $17-23 \mathrm{~Hz}$, the point of sign inversion localizes the same crack tip position, while at $8 \mathrm{~Hz}$ the point is shifted of $0.14 \mathrm{~mm}$.

Considering the horizontal profile passing for the crack tip and parallel to the direction of the crack propagation, the extent of the sign inversion zone passes from 1,30 to $2,9 \mathrm{~mm}$.

- $\quad$ The parameter $T_{2}$ presents absolute values and the area with values above the elastic zone average signal which decrease with the frequency up to $15 \mathrm{~Hz}$. From 15 to $23 \mathrm{~Hz}$ the trend reverses and both the absolute values and the area increase with the frequency, but the trend is not clear due to the high noise value.

- The data obtained for the parameter $\Phi_{2}$ are difficult to interpreter due to the noise. However, it is possible to estimate a common trend which is characterized by a sign inversion ahead the crack tip.

Both the $T_{2}$ and $\Phi_{2}$ maps prove the presence of the crack closure which appears as an increase of both the parameters.

The strong dependence of the results on the frequency could indicate that conductive phenomena interfere with dissipative phenomena which are more important at low frequency. To relate the thermal parameters to the dimensions of the plastic zone around the crack tip, the material thermal-physic characteristics and all the heat sources should be considered, including the crack closure contribution.

Further work will imply the comparison of the experimental results with the study of the thermal behaviour of the Titanium cracked CT sample through a FEM analysis, by simulating all the heat sources involved. Furthermore, a procedure to evaluate the plastic zone extension will be studied by comparing the plastic zone shape and dimension with results obtained with method based both on Digital Image Correlation and theoretical models. 


\section{REFERENCES}

[1] Weertman J. "Theory of fatigue crack growth based on a BCS crack theory with work hardening". Int J. of Fatigue 9, 125-155, 1973.

[2] Klingbeil N.W. "A total dissipated energy theory of fatigue crack growth in ductile solids". Int J. of Fatigue 25(2), pp. 117-128, 2003.

[3] Mazari M., Bouchouicha B., Zemri M., Benguediab M., Ranganathan N. "Fatigue crack propagation analyses based on plastic energy approach", 2008. Computational Materials Science 41, 344-349, 2003).

[4] Ikeda S., Izumi Y, Fine M.E. "Plastic work during fatigue crack propagation in a high strength low alloy steel and in 7050 Al alloy". Eng Fract Mech 9, pp. 123-128,1977.

[5] Yates JR et al. "Quantifying crack tip displacement fields with DIC". Eng Fract Mech, 77, pp. 2063-14, 2010.

[6] Carrascal I., Casado J.A., Diego S., Lacalle R., Cicero S., Álvarez J.A. "Determination of the Paris' law constants by means of infrared thermographic techniques". Polymer Testing 40, pp. 39-45, 2014.

[7] Meneghetti G., Ricotta M."Evaluating the heat energy dissipated in a small volume surrounding the tip of a fatigue crack", Int J. of Fatigue 92 (2), pp. 605-615, 2016.

[8] Palumbo D., De Finis R., Ancona F., Galietti U. "Damage monitoring in fracture mechanics by evaluation of the heat dissipated in the cyclic plastic zone ahead of the crack tip with thermal measurements". Eng Fract Mech 181, pp. 65-75, 2017.

[9] Diaz F.A., Patterson E.A.,Yates R.A. "Some improvements in the analysis of fatigue cracks using thermoelasticity". Int J. of Fatigue 26(4), pp. 365-376, 2004.

[10] F.Ancona, R. De Finis, G. P. Demelio, U. Galietti, D. Palumbo, Study of the plastic behavior around the crack tip by means of thermal methods, Procedia Structural Integrity 2 (2016) 2113-2122

[11] G. Pitarresi, EA. Patterson, A review of the general theory of the thermoelastic stress analysis. The Journal of Strain Analysis for Engineering Design 38(5) (2003) 405-17.

[12] W. J. Wang, J. M. Dulieu-Barton, Q. Li, Assessment of non-adiabatic behaviour in thermoelastic stress analysis of small-scale components. Experimental Mechanics 50 (2010) 449-61.

[13] Enke, NF., Sandor, BI., 1988. Cyclic plasticity analysis by differential infrared thermography. Proceeding of the VII International Congress on Experimental Mechanics, 830-835.

[14] Sakagami, T., Kubo, S., Tamura, E., Nishimura, T., 2005. Identification of plastic-zone based on double frequency lock-in thermographic temperature measurement. International Conference of Fracture ICF 11

[15] Wong, A.K.; Sparrow, J.G.; Dunn, S.A. On the revised theory of the thermoelastic effect. J. Phys. Chem. Solids 1988, 48, 395-400.

[16] Palumbo, D.; Galietti, U. Data correction for thermoelastic stress analysis on titanium components. Exp. Mech. 2016, 56, 451-462.

[17] Connesson, N., Maquin, F., Pierron, F., 2011. Experimental Energy Balance During the First Cycles of Cyclically Loaded Specimens Under the Conventional Yield Stress. Experimental Mechanics, 51, 23-45. 